\title{
Serine/Threonine-Protein Kinase 36
}

National Cancer Institute

\section{Source}

National Cancer Institute. Serine/Threonine-Protein Kinase 36. NCI Thesaurus. Code C150326.

Serine/threonine-protein kinase 36 (1315 aa, 144 kDa) is encoded by the human ST K36 gene. This protein is involved in the induction of GLI zinc-fing er family transcription factor activity. 